\title{
The role of Pyk2 in the CCR7-mediated regulation of metastasis and viability in squamous cell carcinoma of the head and neck cells in vivo and in vitro
}

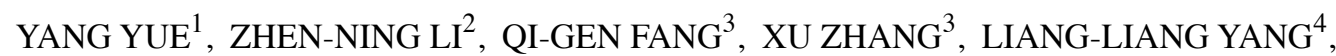 \\ CHANG-FU SUN ${ }^{2}$ and FA-YU LIU ${ }^{2}$
}

\begin{abstract}
${ }^{1}$ Department of Orthodontics, School of Stomatology, China Medical University; ${ }^{2}$ Department of OromaxillofacialHead and Neck Surgery, Department of Oral and Maxillofacial Surgery, School of Stomatology, China Medical University, Shenyang, Liaoning 110002; ${ }^{3}$ Department of Head and Neck, Henan Tumor Hospital, Zhengzhou University, Zhengzhou, Henan 450008; ${ }^{4}$ Department of Oral and Maxillofacial Surgery, The Fourth Affiliated Hospital of China Medical University, Shenyang, Liaoning 110002, P.R. China
\end{abstract}

Received May 31, 2015; Accepted August 13, 2015

DOI: $10.3892 /$ or.2015.4269

\begin{abstract}
In the present study, we aimed to demonstrate whether praline-rich tyrosine kinase-2 (Pyk2) participates in the chemokine receptor 7 (CCR7) downstream signaling network, and to determine the role of this molecule and the related mechanism in the CCR7-mediated regulation of viability and metastasis in vivo and in vitro of squamous cell carcinoma of the head and neck (SCCHN). We constructed the stable Pyk2 related non-kinase (PRNK)-expressing SCCHN cell line, and examined the viability, apoptosis, migration, invasion and adhesion ability in the transfected and untransfected SCCHN cells. An SCCHN tumor model in nude mice was designed and the tumor growth rate was assayed. E-cadherin and vimentin expression was assessed when Pyk2 was inactivated. We found that the stable PRNKexpressing SCCHN cells exhibited low viability, a high rate of apoptosis, low migratory ability, low invasive ability and low adhesion capacity. In the nude mouse body, the tumors
\end{abstract}

Correspondence to: Dr Fa-Yu Liu, Department of Oromaxillofacial-Head and Neck Surgery, Department of Oral and Maxillofacial Surgery, School of Stomatology, China Medical University, 117 Nanjing Bei Jie, Heping, Shenyang, Liaoning 110002, P.R. China

E-mail:1fyhjk@126.com

Abbreviations: Pyk2, praline-rich tyrosine kinase-2; CCR7, chemokine receptor 7; SCCHN, squamous cell carcinoma of the head and neck; PRNK, praline-rich tyrosine kinase-2 related non-kinase; EGFP, enhanced green fluorescent protein; CCL19, c-c motif chemokine 19; ANF, $\alpha$-naphthoflavone; EMT, epithelialmesenchymal transition; DMEM, Dulbecco's modified Eagle's medium; OD, optical density; FITC, fluorescein isothiocyanate

Key words: Pyk2, CCR7, PRNK, metastasis, squamous cell carcinoma of the head and neck formed by these cells grew slowly when compared to the tumor growth in the control group. When Pyk2 was inactivated, CCR7-induced E-cadherin and vimentin expression levels were altered. Thus, Pyk2 is a key downstream signaling molecules of CCR7 in SCCHN, which promotes SCCHN tumorigenesis and progression.

\section{Introduction}

Squamous cell carcinoma of the head and neck (SCCHN), a malignant tumor of epithelial origin, accounts for more than $90 \%$ of all head and neck cancers, with a 5-year survival rate of only $\sim 30-40 \%$ (1). The main reasons for the poor diagnosis of this disease are tumor recurrence and metastasis to lymph nodes. Therefore, a better understanding of the processes involved in its recurrence and metastasis is necessary to enable the development of therapies designed to prevent tumor dissemination.

Chemokines are a group of small structurally related molecules that constitute a superfamily of inducible secreted proinflammatory proteins that are involved in a variety of immune responses (2-5). In has been reported that the CC chemokine CCL19 and its receptor chemokine receptor 7 (CCR7) participate in the metastasis of various types of tumors, such as uveal melanoma, pancreatic ductal adenocarcinoma and gastric cancer, in a manner such as 'lymphocyte homing' (6-8). Our previous studies demonstrated that CCR7 can regulate cell migration and invasion of $\mathrm{SCCHN}$, and this process involves several signaling pathways including PI3K/cdc42, MAPK and JAK2/STAT3 (9-11).

In a previous study, we also found that praline-rich tyrosine kinase-2 (Pyk2) is activated by CCL19-CCR7 interaction in SCCHN cells, and the Pyk2 inhibitor inhibits tumor cell mobility (12). The results suggest that Pyk2 may be involved in the CCR7-mediated regulation of SCCHN cell biological activity. The aim of the present study was to further demonstrate whether Pyk2 participates in the CCR7 downstream 
signaling network, and to determine whether this molecule plays a role in the CCR7-mediated regulation of SCCHN viability and metastasis in vivo and in vivo and the involved mechanisms.

\section{Materials and methods}

Cell lines. PCI-4B and PCI-37B, well-characterized SCCHN cell lines derived from the metastatic lymph node of SCCHN patients, were kindly donated by the University of Pittsburgh Cancer Institute $(13,14)$. The cells were cultured in Dulbecco's modified Eagle's medium (DMEM) (Invitrogen, Carlsbad, CA, USA) containing 10\% fetal bovine serum (FBS) (Gibco, Carlsbad, CA, USA), $100 \mathrm{U} / \mathrm{ml}$ penicillin $\mathrm{G}$ and $100 \mathrm{U} / \mathrm{ml}$ streptomycin.

Reagents and antibodies. CCL19- and CCR7-specific monoclonal antibodies (mouse anti-human CCR7 antibody) were purchased from R\&D Systems (Minneapolis, MN, USA). Anti-Pyk 2 and anti-phospho-Pyk2 were purchased from Santa Cruz Biotechnology (Dallas, TX, USA). Antivimentin and anti-E-cadherin were purchased from Cell Signaling Technology (Danvers, MA, USA). The treatment conditions, such as concentration and time, were drawn from pre-experiments.

CCR7 siRNA and PRNK transfection. The Pyk2 related non-kinase (PRNK) is a recently described isoform of Pyk2 that encodes part of the COOH-terminal domain of Pyk2 and has been proposed to selectively downregulate Pyk2 function (15). Lentiviruses containing the PRNK gene (LV-PRNK-EGFP) or a negative control (Lenti-EGFP) were obtained from Shanghai GeneChem, China. The construction of PRNK recombinant lentiviral vectors was as follows. The GV218 vector (Lenti-EGFP; purchased from Shanghai GeneChem) was linearized with BamHI and AgeI. The PRNK gene cDNA sequence was as described by Xiong et al (15), and synthesized and purchased from Shanghai GeneChem. The cDNA was also digested with BamHI and AgeI, and ligated into the GV218 vector. The constructs were verified by sequencing. The Lenti-EGFP was lentiviruses ligated with EGFP by T4 DNA ligase, 5' exonuclease and Taq polymerase. Viruses were propagated in 293 cells, clonally isolated and titered. SCCHN cells were infected at a matched multiplicity of infection (MOI) of 10. CCR7 siRNA was purchased from Santa Cruz Biotechnology. Transfection of the SCCHN cells with $20 \mathrm{nM}$ of siRNA was carried out using Lipofectamine SiRNAMAX (Invitrogen) for $32 \mathrm{~h}$.

Western blot analysis. Cells were gathered and lysed. Lysates were sonicated for $3 \mathrm{sec}$ and centrifuged at $4^{\circ} \mathrm{C}$ and 14,000 rpm for $30 \mathrm{~min}$. The supernatant was collected for protein quantification using the Bio-Rad protein assay dye reagent (Bio-Rad Laboratories, Richmond, CA, USA). Fifty micrograms of protein were size-fractionated through a $10 \%$ SDS-PAGE gel and transferred onto nitrocellulose filters. The filters were blocked [5\% non-fat dry milk, 0.1\% Triton X-100, $150 \mathrm{mM}$ $\mathrm{NaCl}, 50 \mathrm{mM}$ Tris [tris(hydroxymethyl)aminomethane] ( $\mathrm{pH} 7.5)]$ and incubated with the primary antibody, which was diluted to a ratio of 1:1,000. Nitrocellulose filters were incubated with horseradish peroxidase-conjugated secondary antibodies. Bands were visualized using the enhanced chemiluminescence system (Amersham Pharmacia Biotech, Piscataway, NJ, USA) and quantified by scanning densitometry using FluorChem v2.0 software (Alpha Innotech Corporation, San Leandro, CA, USA).

Migration assay. Disposable 24-well Transwell inserts with a $80-\mu \mathrm{m}$ pore size were run in triplicate in DMEM with $0.5 \%(\mathrm{w} / \mathrm{v}) \mathrm{BSA}$. CCL19 was added to the lower chamber at a concentration of $500 \mathrm{ng} / \mathrm{ml}$. Cell suspensions $\left(2 \times 10^{5}\right)$ were placed in the top of the inserts. After $24 \mathrm{~h}$ of incubation, the cells on the upper surface of the inserts were removed with a cell harvester, and the membrane was washed with medium. Cells that penetrated the membrane were fixed with ice-cold methanol, stained with $0.5 \%$ crystal violet, photographed and counted under a microscope. Migration index was calculated based on the control involving random migration.

Matrigel invasion assay. Cell invasion was quantified in vitro using Matrigel-coated semi-permeable, modified inserts with a pore size of $8-\mu \mathrm{m}$. The analysis of the Matrigel invasion assay was performed as described in the migration assay incubated with CCL19 for $36 \mathrm{~h}$. Invasion index was calculated based on the control involving random invasion.

Viability assay. The viability assay is a colorimeter assay that relies on the ability of viable cells to convert a soluble tetrazolium salt, MTT, into a quantifiable insoluble formazan precipitate (yellow to purple color change). Cell death correlates with reduced purple precipitate formation. In brief, SCCHN cells were plated into a 96-well plate at an initial density of $5 \times 10^{4}$ cells/well. Confluent cells (70-80\%) were serum starved for $24 \mathrm{~h}$, and then treated with CCL19. Media were discarded, and $20 \mu \mathrm{l}$ of MTT $(5 \mu \mathrm{g} / \mathrm{ml}$ in PBS) was added for $4 \mathrm{~h}$. DMSO $(200 \mu \mathrm{l})$ was then added to each well, and the plate was incubated at $37^{\circ} \mathrm{C}$ in a $5 \% \mathrm{CO}_{2}$ atmosphere for $1 \mathrm{~h}$ to lyse all of the cells, and the media were collected for measurement. The optical density (OD) was read by a spectrometer (Tecan, Männedorf, Switzerland) at $490 \mathrm{~nm}$. The percentage of cell viability was calculated as follows: Viability percentage $(\%)=(\mathrm{OD}$ of treatment group $) /(\mathrm{OD}$ of control group $) \times 100 \%$.

Adhesion assay. For the assay, 96-well plates (Corning Costar, Cambridge, MA, USA) were coated with fibronectin or left uncoated, and incubated at room temperature overnight. SCCHN cells were plated in an initial density of $1 \times 10^{4}$ cells/well, and inbubated at $37^{\circ} \mathrm{C}$ for $1.5 \mathrm{~h}$, and then washed with phosphate-buffered saline (PBS) three times. MTT $(20 \mu 1,5 \mu \mathrm{g} / \mathrm{ml})$ was added for $4 \mathrm{~h}$, and then dimethylsulphoxide (DMSO; Sigma, USA) was added to each well to lyse all cells for $1 \mathrm{~h}$ at $37^{\circ} \mathrm{C}$ at $5 \% \mathrm{CO}_{2}$. The OD was read on a spectrometer (Tecan) through a 490-nm filter. For each experimental condition 8 wells were analyzed in parallel. Adhesion index $(\%)=[($ fibronectin conditions cell OD/control conditions cell OD) - 1] x $100 \%$.

Apoptosis assay. To determine the role of the Pyk2 pathway in apoptosis, Annexin V and propidium iodide (PI) staining was performed. Briefly, the transfected cells were pretreated and 

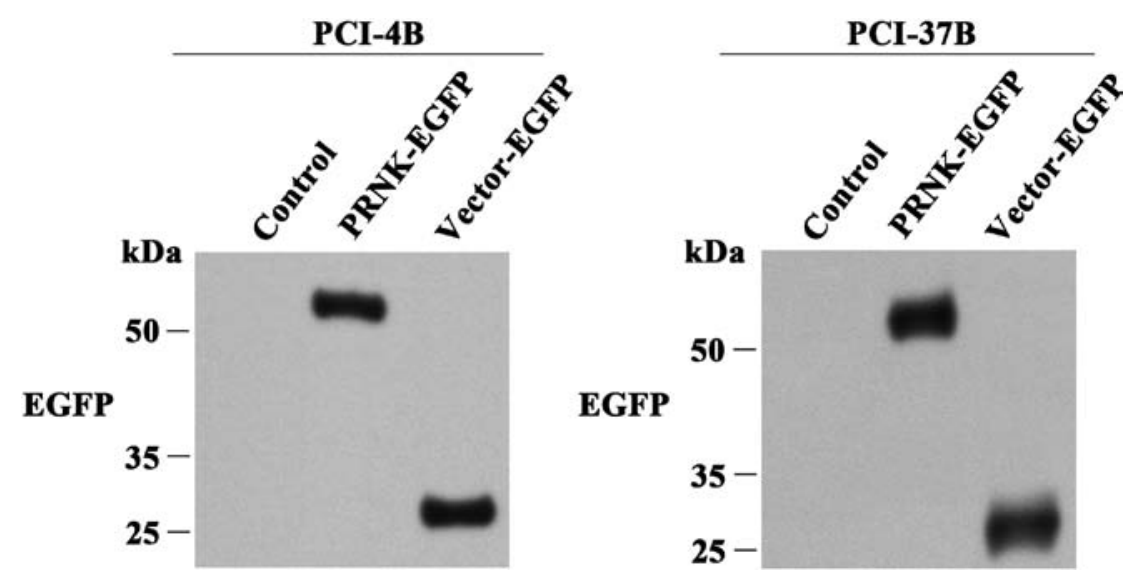

Figure 1. Western blot analysis of EGFP expression. PCI-4B and PCI-37B cells were transfected with LV-EGFP with/without PRNK and EGFP expression was assessed. The results are representative of three independent experiments.

harvested at the indicated time points. Cells were stained with fluorescein isothiocyanate (FITC)-conjugated Annexin V and PI according to the manufacturer's recommendation. In these experiments, the cells that were apoptotic were those that were Annexin V-positive/PI-negative. Samples were analyzed using a FACSCalibur flow cytometer and CellQuest software (BD, Sparks, MD, USA).

Xenograft studies of nude mice. BALB/c athymic nude mice (4 weeks of age) were maintained under standard laboratory conditions on a 12-h light-dark cycle and given access to sterilized food and water ad libitum under a specific pathogen-free environment. For the subcutaneous injection, the cells $\left(1 \times 10^{6}\right)$ were suspended in $200 \mu \mathrm{l}$ PBS and then inoculated subcutaneously into the right breast region of the BALB/c nude mice. Each group consisted of six nude mice. Two-dimensional measurements were taken with an electronic caliper, and the tumor volume in $\mathrm{mm}^{3}$ was calculated using the formula: Volume $=\mathrm{ax} \mathrm{b}^{2} \times 0.52$, where $\mathrm{a}$ is the longest diameter; $\mathrm{b}$ is the shortest diameter. At the terminal point when the mice were sacrificed, the tumors were fixed in $10 \%$ formalin overnight and subjected to routine histological examination. All of the animals were treated according to the protocols approved by the Institutional Animal Care and Use Committee of China Medical University.

Statistical analysis. Data are expressed as the mean \pm standard deviation (SD) of repeated assays. Significant differences between the two groups were evaluated using an unpaired Student's t-test. A p-value $<0.05$ was considered to indicate a statistically significant result. All statistical analyses were performed with software SPSS 11.0 (SPSS, Inc., Chicago, IL, USA).

\section{Results}

PRNK transfection and its role in Pyk2. PRNK is the $\mathrm{C}$-terminal non-kinase region of Pyk2. It is characterized as a splice variant form of Pyk2. PRNK will interact with several of the Pyk2-binding partners due to sequence similarity with Pyk2. Therefore, it has been reported as a dominant-negative regulator to prevent Pyk2 binding with its partners for

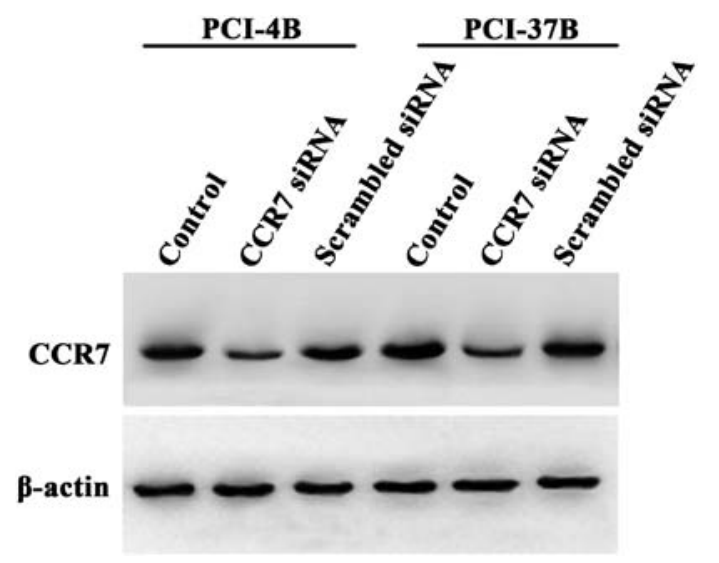

Figure 2. Western blot analysis of CCR7 expression. PCI-4B and PCI-37B cells were transfected with CCR7 siRNA or scrambled siRNA and the protein expression of CCR7 was assessed. The results are representative of three independent experiments.

signaling pathway activation (15). In the present study, we constructed the lentivirus shuttle vector merged with EGFP for PRNK transfection, and examined the EGFP expression which represented the efficacy of the PRNK transfection. As shown in Fig. 1, in the control group, EGFP expression was not observed. In the LV (vector)-EGFP control group, EGFP expression was observed at $27 \mathrm{kDa}$, indicating that the transfection was effective. In the LV-PRNK-EGFP group, EGFP expression was also observed, but it was located at $\sim 52-54 \mathrm{kDa}$, suggesting it is a compound of PRNK and EGFP.

In a previous study, we observed that the phosphorylation of Pyk 2 was inhibited by the CCR7 monoclonal antibody (12). In the present study, we transiently transfected CCR7 siRNA into SCCHN cells to silence CCR7 (Fig. 2) (16), and used CCL19 to induce the CCR7 downstream signaling pathway. The results showed that CCL19 induced the phosphorylation of Pyk2, and CCR7 siRNA significantly reduced this phosphorylation, which had a significant difference compare to the CCL19 and scramble siRNA groups (Fig. 3). These results demonstrated that Pyk2 is a downstream signaling molecule of CCR7. In the LV-EGFP group, the Pyk2 phosphorylation was similar to the CCL19 group, and in the LV-PRNK-EGFP group, the Pyk2 

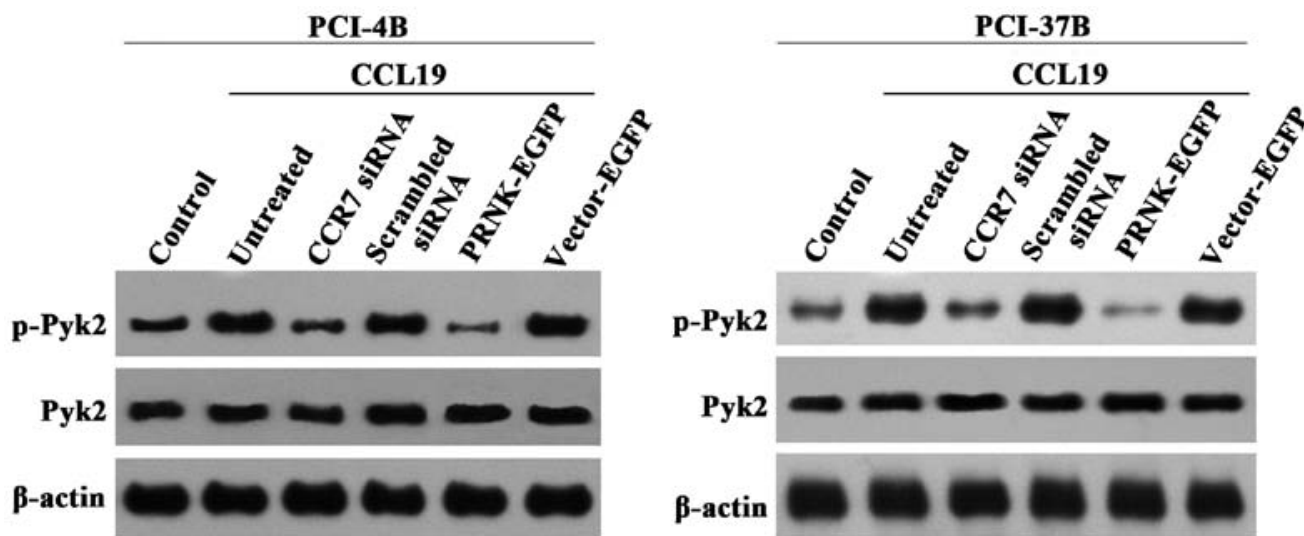

Figure 3. Western blot analysis of Pyk2 expression. PCI-4B and PCI-37B cells were transfected with CCR7 siRNA, scrambled siRNA, LV (vector)-PRNKEGFP and LV-EGFP, treated with CCL19 $(200 \mathrm{ng} / \mathrm{ml})$ for $5 \mathrm{~min}$ and then the phosphorylation or total protein expression of Pyk 2 was assessed. The results are representative of three independent experiments.
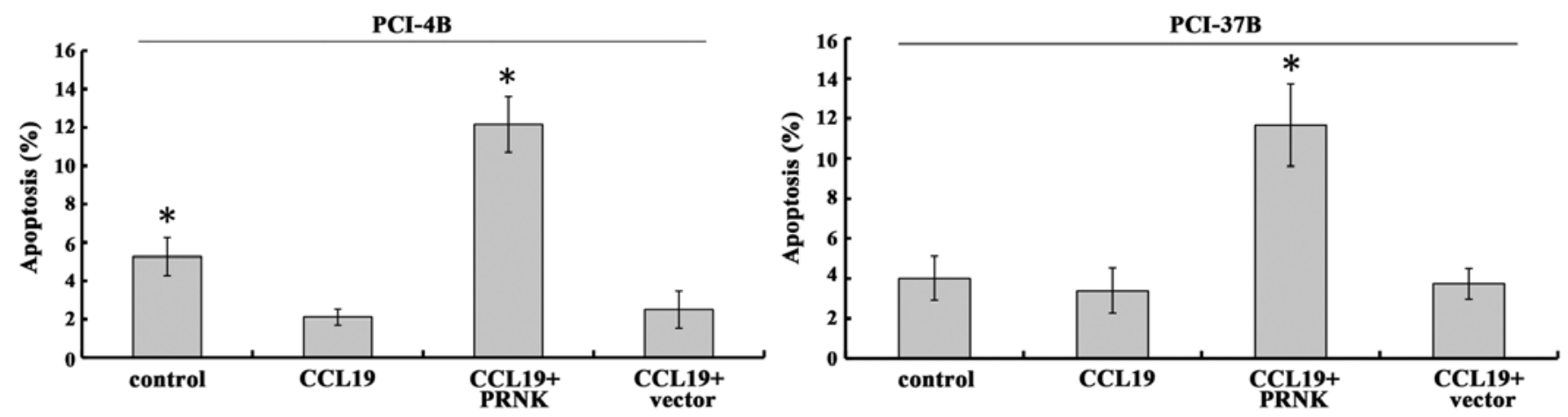

Figure 4. The role of Pyk2 in regulating apoptosis in the SCCHN cells. The PCI-4B and PCI-37B cells, PRNK- or vector-transfected PCI-4B and PCI-37B cells were treated with CCL19 $(400 \mathrm{ng} / \mathrm{ml})$ for $6 \mathrm{~h}$ and then apoptosis was assessed by Annexin V/PI staining and flow cytometry. The results are representative of three independent experiments. " $\mathrm{p}<0.05$ compared to the CCL19 group.
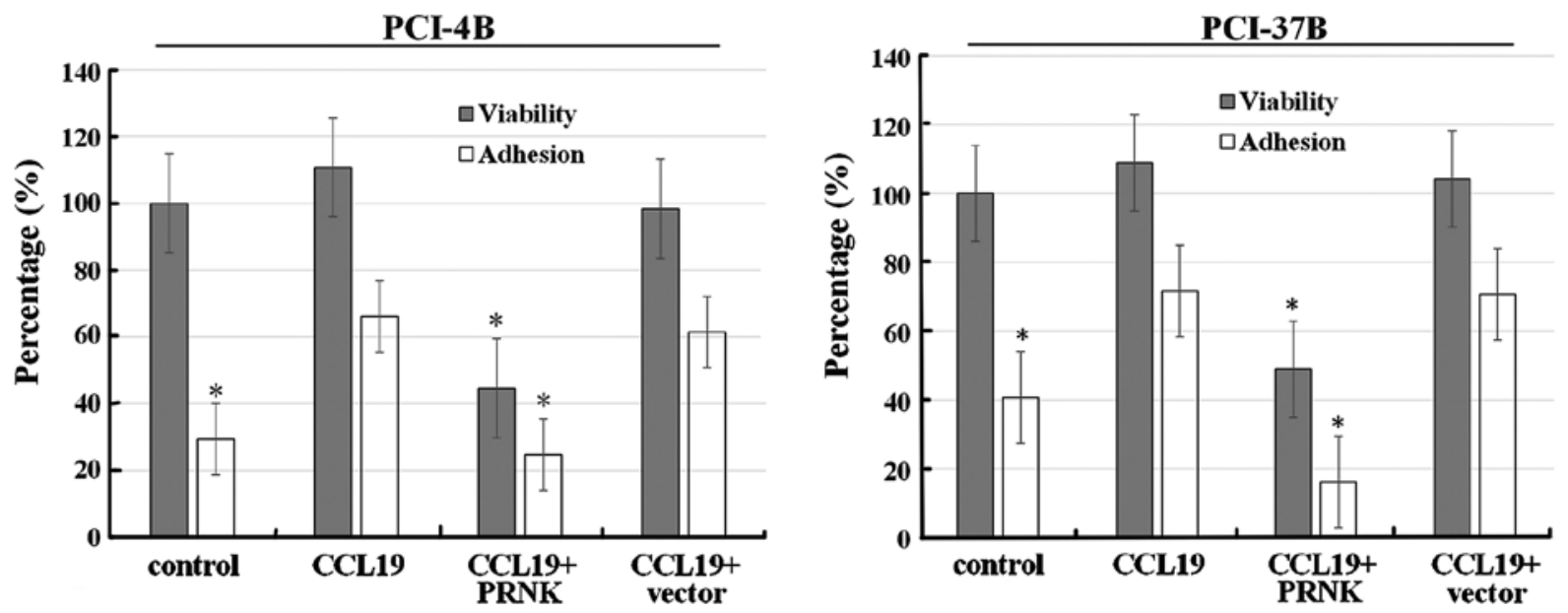

Figure 5. The role of Pyk2 in regulating viability and adhesion in the SCCHN cells. The PCI-4B and PCI-37B cells, PRNK- or vector-transfected PCI-4B and PCI-37B cells were treated with CCL19 $(400 \mathrm{ng} / \mathrm{ml})$ for $30 \mathrm{~min}$ and cell adhesion was assessed, while at $6 \mathrm{~h}$ cell viability was assessed by MTT assay. The results are representative of three independent experiments. ${ }^{*} \mathrm{p}<0.05$ compared to the CCL19 group.

phosphorylation was significantly reduced. In contrast, the total Pyk2 protein expression had no change in these groups. The results demonstrated that PRNK is a competitive inhibitor of Pyk 2 constitutional activation, which will not interfere with Pyk2 expression.
Pyk2 regulates CCL19-induced SCCHN cell viability, migration, invasion and adhesion. Our previous study used cisplatin to induce cell death and demonstrated that CCL19 can promote PCI-4B and PCI-37B cell viability, migration and invasion, and this function can be blocked by CCR7 monoclonal 

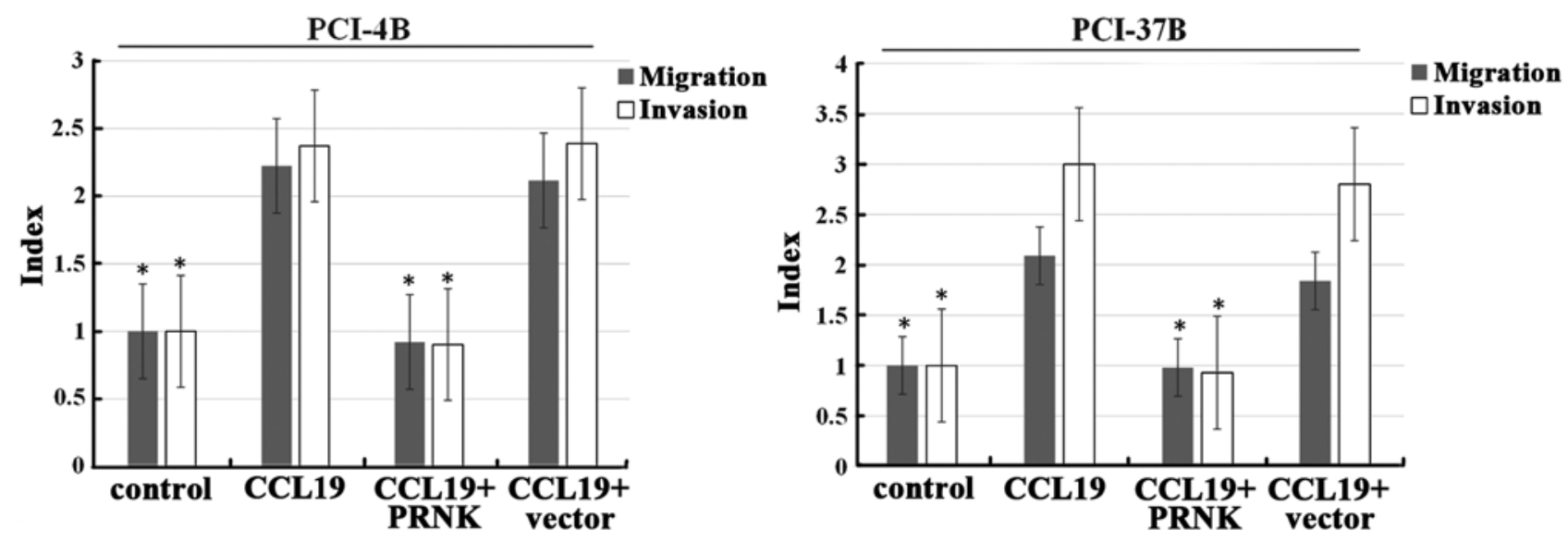

Figure 6. The role of Pyk2 in regulating cell migration and invasion in the SCCHN cells. The PCI-4B and PCI-37B cells, PRNK- or vector-transfected PCI-4B and PCI-37B cells were treated with CCL19 $(500 \mathrm{ng} / \mathrm{ml})$ for $24 \mathrm{~h}$ and cell migration was assessed, while at $36 \mathrm{~h}$ cell invasion was assessed. The results are representative of three independent experiments. "p $<0.05$ compared to the CCL19 group.
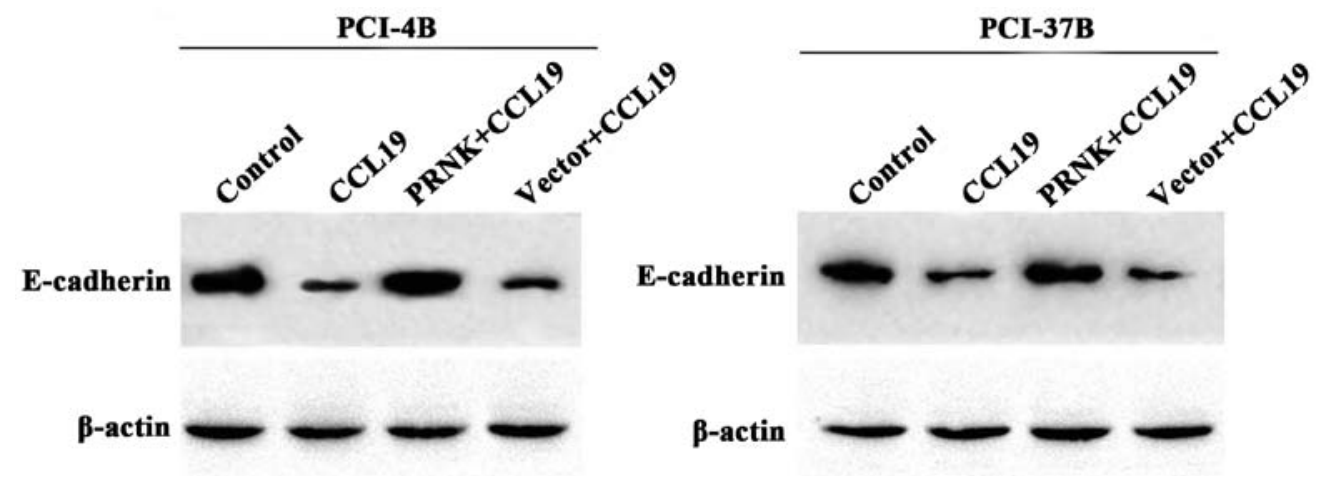

Figure 7. The role of Pyk2 in CCL19-induced E-cadherin expression in SCCHN cells. The PCI-4B and PCI-37B cells, PRNK- or vector-transfected PCI-4B and PCI-37B cells were treated with CCL19 $(200 \mathrm{ng} / \mathrm{ml})$ for $30 \mathrm{~min}$, and then E-cadherin expression was assessed by western blotting. The results are representative of three independent experiments.

antibody $(10,11,17-20)$. In the present study, we examined whether Pyk2 is involved in regulating these bioactivities.

In the PCI-4B and PCI-37B cell lines, treatment of CCL19 protected the cells from apoptosis (Fig. 4) (19). Yet, in the PRNK-transfected PCI-4B and PCI-37B cells, the role of CCL19 was blocked, and the apoptosis was significantly increased 2-3 times in the PCI-4B and PCI-37B cell lines. As a control, the vector transfection group had no significant change. This indicates that PRNK promotes SCCHN cell apoptosis.

As shown in Fig. 5, following CCL19 treatment, the viability of the PCI-4B and the PCI-37B cells was unaffected $(\sim 100-110 \%)$, while the viability of the PRNK-transfected PCI-4B and PCI-37B cells was reduced to less than 50\%; the difference was significant. As a control, the vector transfection group had no significant change. The results indicated that PRNK can induce cell death.

We also examined the adhesive ability of the SCCHN cells (Fig. 5). Following CCL19 pretreatment, the adhesion index of the PCI-4B and the PCI-37B cells increased from $30-40 \%$ of control condition to $\sim 70 \%$, suggesting that CCL19 promotes SCCHN cell adhesion to fibronectin. In the stable PRNKexpressing PCI-4B and PCI-37B cells, CCL19 played no role in this adhesion induction, and the adhesion index decreased to the control level. This indicates that the interaction between CCL19 and CCR7-induced adhesion was Pyk2-dependent.

A migration assay was next carried out. Following CCL19 induction, the cell migration index of the PCI-4B and PCI-37B cells was $>2$ times that of the control group (Fig. 6). PRNK transfection significantly blocked the effect of CCL19 in both cell lines, leading to decreased cell migration to almost baseline level. The invasion index was the same as the migration index, and CCL19-induced invasion was significantly blocked by PRNK transfection. As a control, the vector transfection group had no significant change in migration and invasion.

Pyk 2 mediates the expression of E-cadherin and vimentin induced by CCR7. E-cadherin and vimentin are known to be associated with epithelial-mesenchymal transition (EMT), which is a key point in tumor progression and generally participates in cell migration and invasion. Our previous results demonstrated that CCL19-treated PCI-4B and PCI-37B cells exhibited a significant increase in the level of vimentin protein and a significant decrease in the level of E-cadherin (10). In the present study, we examined the PRNK-transfected PCI-4B and PCI-37B cells, and found that, with the Pyk2 inaction the CCL19-induced low E-cadherin expression was significantly 


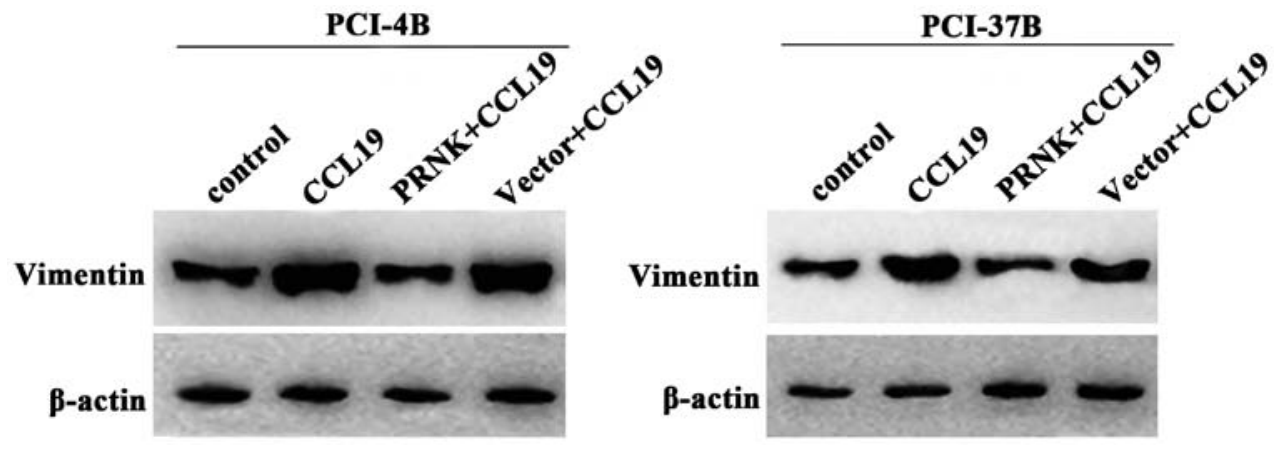

Figure 8. The role of Pyk2 in CCL19-induced vimentin expression in the SCCHN cells. The PCI-4B and PCI-37B cells, PRNK- or vector-transfected PCI-4B and PCI-37B cells were treated with CCL19 $(200 \mathrm{ng} / \mathrm{ml})$ for $30 \mathrm{~min}$ and then the vimentin expression was assessed by western blotting. The results are representative of three independent experiments.
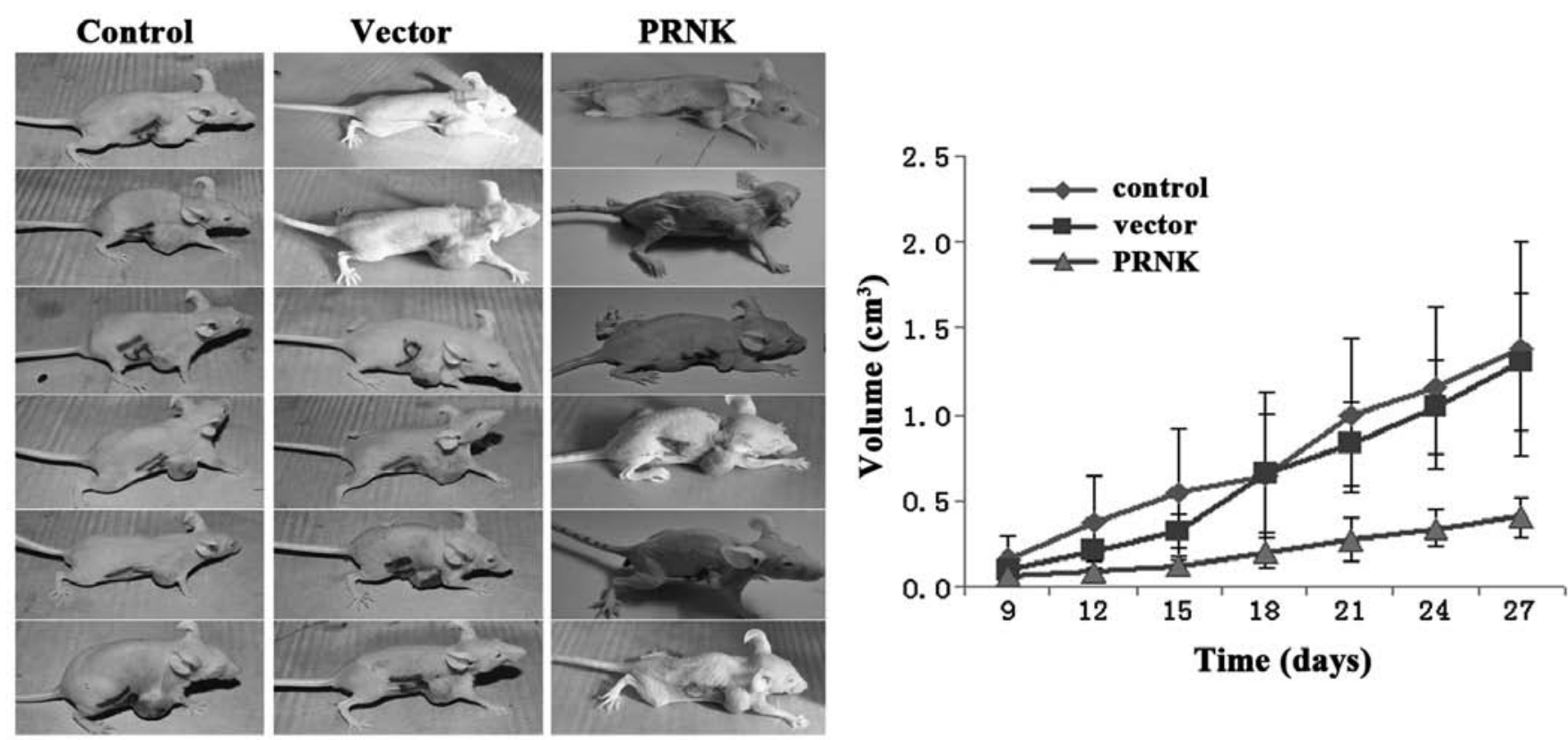

Figure 9. Tumor model in nude mice. BALB/c athymic nude mice were subcutaneously injected with SCCHN cells $\left(1 \times 10^{6}\right)$ in the right breast region, and the tumor volume was calculated at different times.

increased, and the CCL19-induced high vimentin expression was also significantly decreased (Figs. 7 and 8 ). The results supported that the CCR7-mediated E-cadherin and vimentin expression levels in the SCCHN cells were Pyk2-dependent.

Pyk2 inactivation inhibits SCCHN tumorigenesis in vivo. The subcutaneous model of human SCCHN in nude mice was used to assess the role of Pyk2 in tumorigenesis in vivo. The stable PRNK-expressing PCI-37B and control vector-transfected PCI-37B cells were subcutaneously injected into the breast of male nude mice. Tumor growth curves were obtained. As shown in Fig. 9, after injection, the PCI-37B cells developed into a tumor mass on day 9 and grew for 27 days. The tumors formed by the control vector-transfected PCI-37B cells exhibited the same growth rate as the control PCI-37B cell group, and reached a volume of $\sim 1.5 \mathrm{~cm}^{3}$ on day 27 . However, the stable PRNK-expressing PCI-37B cells grew slowly in the bodies of the nude mice, and on day 27 , only formed small tumors, which were no more than $0.5 \mathrm{~cm}^{3}$. There was a signifi- cant difference in tumor size between the cells expressing PRNK or the cells that did not. This finding indicates that Pyk2 activity promotes SCCHN tumorigenesis in vivo.

\section{Discussion}

Pyk2 is a non-receptor tyrosine kinase which is believed to play a role in transducing extracellular matrix (ECM)-derived survival signals into cells. The functions of Pyk 2 are linked to autophosphorylation of their specific tyrosine residues, and then association with different signaling proteins which mediate activation of downstream targets. Thus, modulation of Pyk2 autophosphorylation may affect several intracellular pathways and may participate in a variety of pathological settings (21). The Pyk2 related non-kinase (PRNK) is a recently described isoform of Pyk2 that encodes part of the $\mathrm{COOH}$-terminal domain of Pyk2 (22). As a splice variant form of Pyk2, PRNK will interact with some of the Pyk2-binding partners due to sequence similarity with Pyk2. Therefore, it has been reported 
as a dominant-negative regulator to prevent Pyk2 binding with its partners for signaling pathway activation and PRNK has been used in numerous studies (23).

In the present study, we constructed stable PRNKexpressing SCCHN cells, and demonstrated the transfection efficacy by examining EGFP. CCR7 siRNA, which induces CCR7 silencing (16), blocked the Pyk2 phosphorylation induced by CCL19 as well as PRNK, and had no effect on Pyk2 expression. This indicated that Pyk2 phosphorylation was CCR7 activation-dependent. This finding was similar to the results of a previous study (12).

Squamous cell carcinoma of the head and neck exhibits metastatic potential. Metastasis involves the separation of cells from the primary tumor, migration into the extracellular matrix, blood vessel invasion, adhesion to endothelium and extravasation and growth in a secondary organ (24). Pyk2 has been reported to widely participate in this process. In hepatocellular carcinoma, overexpression of Pyk 2 resulted in an upregulation of colony formation, adhesion toward laminin, cell motility and wound recovery (25). In malignant glioma, overexpression of Pyk2 stimulated cell migration (22). Doxorubicin-resistant MCF-7 (MCF-7/Dox) breast cancer cells expressed constitutively active forms of Pyk2 compared with the expression in parental MCF-7 cells. $\alpha$-naphthoflavone (ANF) is a wellknown chemopreventive agent; its anticancer properties were found to function by inhibition of clonogenic cell survival via de-phosphorylation of FAK, Pyk2 and EGF-induced Akt in MCF-7/Dox cells and tumor xenografts (26). In a previous study, following induction with CCL19, SCCHN cells exhibited high viability, a low rate of apoptosis, high migratory ability, high invasive ability and adhesion capability $(9-11,27,28)$. In the present study, we found that the cellular functions mediated by CCL19 were abrogated by PRNK, which competitively inhibited Pyk2 activity, suggesting that Pyk2 widely participates in the CCR7-mediated SCCHN cell survival and metastatic ability. In order to demonstrate this result in vivo, a nude mouse tumor-bearing model was performed. We found that stable PRNK expression in SCCHN cells significantly inhibited tumor growth in nude mice, which supported the in vitro results.

The activity of Pyk2 regulates physiological processes by many target proteins. Our previous study demonstrated that the Pyk2 inhibitor Tyrphostin A9 blocked CCL19-induced STAT3 phosphorylation, and this STAT3 phosphorylation was involved in the migration and invasion of SCCHN cells (11). CCL19 was also found to decrease E-cadherin expression and increase vimentin expression in SCCHN cells (10). The constitutive expression of E-cadherin in both normal and cancer cells may help to maintain adherence junctions and subsequently decrease the capacity of cells to invade or migrate through the extracellular matrix $(29,30)$. The important role that EMT plays in tumor progression is known to partly depend on dismantling cadherin-mediated cell-cell junctions (31). Vimentin, the major intermediate filament (IF) protein of mesenchymal cells, is also associated with EMT. In the present study, when Pyk2 was inactivated, low E-cadherin expression was increased and high vimentin expression was decreased, although with CCL19 induction. Therefore, we presumed that E-cadherin and vimentin are downstream target molecules of CCR7-Pyk2, and this signaling pathway may participate in carcinoma cell growth, separation from the primary tumor, migration into the extracellular matrix, invasion into the lymphatic system, adherence to the endothelium and thus achieve metastasis.

Taken together, based on our in vivo and in vitro study, our results support the conclusion that Pyk2 plays a key role in CCR7-mediated regulation of SCCHN cell metastasis and viability. The present study will aid in the understanding of the mechanism involved in the regulation of CCR7 in SCCHN tumorigenesis and progression. Our ultimate aim is to develop therapeutic methods according to our basic research in the near future.

\section{Acknowledgements}

This study was supported by grants from the National Natural Science Foundation of China (no. 81372877), the National Young Scholars Science Foundation of China (no. 81102058), the Foundation of Education Bureau of Liaoning Province (nos. 2009A755 and L2014317), the Public Welfare Fund Project for Science of Liaoning Province (no. 2011002001), the Natural Science Foundation of Liaoning Province (no. 2014021096), and the Excellent Talent Fund Project of Higher Education of Liaoning Province (LJQ2014087).

\section{References}

1. Greenlee RT, Hill-Harmon MB, Murray T and Thun M: Cancer statistics, 2001. CA Cancer J Clin 51: 15-36, 2001.

2. Butcher EC, Williams M, Youngman K, Rott L and Briskin M: Lymphocyte trafficking and regional immunity. Adv Immunol 72: 209-253, 1999.

3. Campbell JJ and Butcher EC: Chemokines in tissue-specific and microenvironment-specific lymphocyte homing. Curr Opin Immunol 12: 336-341, 2000.

4. Morales J, Homey B, Vicari AP, Hudak S, Oldham E, Hedrick J, Orozco R, Copeland NG, Jenkins NA, McEvoy LM, et al: CTACK, a skin-associated chemokine that preferentially attracts skin-homing memory T cells. Proc Natl Acad Sci USA 96: 14470-14475, 1999.

5. Zlotnik A and Yoshie O: Chemokines: A new classification system and their role in immunity. Immunity 12: 121-127, 2000.

6. van den Bosch T, Koopmans AE, Vaarwater J, van den Berg M, de Klein A and Verdijk RM: Chemokine receptor CCR7 expression predicts poor outcome in uveal melanoma and relates to liver metastasis whereas expression of CXCR4 is not of clinical relevance. Invest Ophthalmol Vis Sci 54: 7354-7361, 2013.

7. Guo J, Lou W, Ji Y and Zhang S: Effect of CCR7, CXCR4 and VEGF-C on the lymph node metastasis of human pancreatic ductal adenocarcinoma. Oncol Lett 5: 1572-1578, 2013.

8. Wang WN, Chen Y, Zhang YD and $\mathrm{Hu}$ TH: The regulatory mechanism of CCR7 gene expression and its involvement in the metastasis and progression of gastric cancer. Tumour Biol 34: 1865-1871, 2013.

9. Zhao ZJ, Liu FY, Li P, Ding X, Zong ZH and Sun CF: CCL19induced chemokine receptor 7 activates the phosphoinositide-3 kinase-mediated invasive pathway through Cdc42 in metastatic squamous cell carcinoma of the head and neck. Oncol Rep 25: 729-737, 2011.

10. Liu FY, Safdar J, Li ZN, Fang QG, Zhang X, Xu ZF and Sun CF: CCR7 regulates cell migration and invasion through MAPKs in metastatic squamous cell carcinoma of head and neck. Int J Oncol 45: 2502-2510, 2014.

11. Liu FY, Safdar J, Li ZN, Fang QG, Zhang X, Xu ZF and Sun CF: CCR7 regulates cell migration and invasion through JAK2/STAT3 in metastatic squamous cell carcinoma of the head and neck. Biomed Res Int 2014: 415375, 2014.

12. Yang L, Liu F, Xu Z, Guo N, Zheng X and Sun C: Chemokine receptor 7 via proline-rich tyrosine kinase-2 upregulates the chemotaxis and migration ability of squamous cell carcinoma of the head and neck. Oncol Rep 28: 1659-1664, 2012. 
13. Wang J,Zhang X, Thomas SM, Grandis JR, Wells A, Chen ZG and Ferris RL: Chemokine receptor 7 activates phosphoinositide- 3 kinase-mediated invasive and prosurvival pathways in head and neck cancer cells independent of EGFR. Oncogene 24: 5897-5904, 2005.

14. Mburu YK, Wang J, Wood MA, Walker WH and Ferris RL: CCR7 mediates inflammation-associated tumor progression. Immunol Res 36: 61-72, 2006.

15. Xiong WC, Macklem $M$ and Parsons JT: Expression and characterization of splice variants of PYK2, a focal adhesion kinase-related protein. J Cell Sci 111: 1981-1991, 1998.

16. Zhao ZJ, Liu FY and Sun CF: Effect of chemokine receptor 7 small interfering RNA on proliferation and invasion of squamous cell carcinoma of head and neck. Zhonghua Kou Qiang Yi Xue Za Zhi 44: 5-10, 2009 (In Chinese).

17. Liu FY, Zhao ZJ, Li P, Ding X, Guo N, Yang LL, Zong ZH and Sun CF: NF- $\kappa B$ participates in chemokine receptor 7-mediated cell survival in metastatic squamous cell carcinoma of the head and neck. Oncol Rep 25: 383-391, 2011.

18. Liu FY, Zhao ZJ, Li P, Ding X and Sun CF: The effect of CCL19 on the viability of head and neck squamous cancer cells. Shanghai Kou Qiang Yi Xue 19: 158-161, 2010 (In Chinese)

19. Liu FY, Zhao ZJ, Li P, Ding X, Zong ZH and Sun CF: Mammalian target of rapamycin (mTOR) is involved in the survival of cells mediated by chemokine receptor 7 through PI3K/Akt in metastatic squamous cell carcinoma of the head and neck. Br J Oral Maxillofac Surg 48: 291-296, 2010.

20. Guo N, Liu F, Yang L, Huang J, Ding X and Sun C: Chemokine receptor 7 enhances cell chemotaxis and migration of metastatic squamous cell carcinoma of head and neck through activation of matrix metalloproteinase-9. Oncol Rep 32: 794-800, 2014.

21. Ziemka-Nalecz M, Jaworska J, Sypecka J and Zalewska T: OGD induced modification of FAK-and PYK2-coupled pathways in organotypic hippocampal slice cultures. Brain Res 1606: 21-33, 2015.

22. Lipinski CA, Tran NL, Bay C, Kloss J, McDonough WS, Beaudry C, Berens ME and Loftus JC: Differential role of proline-rich tyrosine kinase 2 and focal adhesion kinase in determining glioblastoma migration and proliferation. Mol Cancer Res 1: 323-332, 2003
23. Sun CK, Ng KT, Lim ZX, Cheng Q, Lo CM, Poon RT, Man K, Wong N and Fan ST: Proline-rich tyrosine kinase 2 (Pyk2) promotes cell motility of hepatocellular carcinoma through induction of epithelial to mesenchymal transition. PLoS One 6: e18878, 2011.

24. Liotta LA, Steeg PS and Stetler-Stevenson WG: Cancer metastasis and angiogenesis: An imbalance of positive and negative regulation. Cell 64: 327-336, 1991.

25. Sun CK, Man K, Ng KT, Ho JW, Lim ZX, Cheng Q, Lo CM, Poon RT and Fan ST: Proline-rich tyrosine kinase 2 (Pyk2) promotes proliferation and invasiveness of hepatocellular carcinoma cells through c-Src/ERK activation. Carcinogenesis 29: 2096-2105, 2008

26. Datta A, Bhasin N, Kim H, Ranjan M, Rider B, Abd Elmageed ZY, Mondal D, Agrawal KC and Abdel-Mageed AB: Selective targeting of FAK-Pyk2 axis by alpha-naphthoflavone abrogates doxorubicin resistance in breast cancer cells. Cancer Lett 362: $25-35,2015$

27. Li P, Liu F, Sun L, Zhao Z, Ding X, Shang D, Xu Z and Sun C: Chemokine receptor 7 promotes cell migration and adhesion in metastatic squamous cell carcinoma of the head and neck by activating integrin $\alpha v \beta 3$. Int J Mol Med 27: 679-687, 2011.

28. Li P, Zhao ZJ, Liu FY, Sun LY, Ding X, Zhang WZ, Shang DH and Sun CF: The chemokine receptor 7 regulates cell adhesion and migration via $\beta 1$ integrin in metastatic squamous cell carcinoma of the head and neck. Oncol Rep 24: 989-995, 2010.

29. Vleminckx K, Vakaet L Jr, Mareel M, Fiers W and van Roy F: Genetic manipulation of E-cadherin expression by epithelial tumor cells reveals an invasion suppressor role. Cell 66: 107-119, 1991.

30. Frixen UH, Behrens J, Sachs M, Eberle G, Voss B, Warda A, Löchner D and Birchmeier W: E-cadherin-mediated cell-cell adhesion prevents invasiveness of human carcinoma cells. J Cell Biol 113: 173-185, 1991.

31. Savagner P: Leaving the neighborhood: Molecular mechanisms involved during epithelial-mesenchymal transition. Bioessays 23: 912-923, 2001. 